Review Article

\title{
반얼굴연축에서 미세혈관감압 수술중 신경계감시
}

\section{용상열*}

원주세브란스기독병원 재활의학과, 연세대학교 원주의과대학

\section{Intraoperative neurophysiologic monitoring in microvascular decompression for hemifacial spasm}

\author{
Sang Yeol Yong* \\ Department of Rehabilitation Medicine, Wonju Severance Christian Hospital, Yonsei University Wonju College of Medicine, \\ Wonju, Korea
}

\begin{abstract}
The majority of primary hemifacial spasm (HFS) patients are related to a vascular compression of the facial nerve root at its entry zone. Non-surgical treatment methods of HFS are anti-epileptic drugs and local botulinum toxin injection, but their outcomes are often not satisfactory in relieving symptoms. Microvascular decompression (MVD) is a surgical treatment method of HFS. Nowadays, intraoperative neurophysiologic monitoring is being used during MVD for two main objectives. The first and main objective is to prevent and reduce the risk of hearing impairment. Brainstem auditory evoked potentials (BAEPs) is monitored during surgery, to monitor the cranial nerve VIII and determine hearing sense preservation. Researches on BAEPs has enabled near real-time and safe monitoring, as warning indicators and advanced techniques to get results faster has been discovered. The second objective is to optimize the effectiveness of surgery. Despite the surgery, some patients had persisted symptoms or recurrence of spasm for some reasons, in few cases. The lateral spread response (LSR) monitoring is performed to guide the operator during surgery. LSR shows modest sensitivity. Nevertheless, it shows high specificity in predicting the symptom-free status after MVD. Low sensitivity precluded the use of the LSR monitoring as an exclusive solitary diagnostic modality. The disappearance of LSR during MVD appears to be not effectively in predicting long-term outcomes, but it demonstrated limited prognostic values in predicting favorable short-term outcomes. These results implements that, intraoperative monitoring of LSR during MVD can provide considerable value for investigating the adequacy of MVD before cranial bone closure.
\end{abstract}

Keywords: hemifacial spasm; intraoperative neurophysiological monitoring; microvascular decompression surgery

\section{Introduction}

Hemifacial spasm (HFS) is a rare neurological disorder that causes irregular involuntary muscle contraction of the face. The symptoms usually begin with orbicularis oculi muscle spasm and then spread to other muscles innervated by ipsilateral facial nerve. This disorder is not a life-threatening, but it leads to profound diminution in quality of life and social withdrawal. The exact mechanism is remains unclear, but the primary cause of this disorder is expected as vas- cular compression of the facial nerve root at its entry zone which can be evidenced with high resolution MRI. There are surgical and non-surgical treatment methods in MVD. Non-surgical treatment methods of HFS are anti-epileptic drugs and local botulinum toxin injection, but their outcomes are often not satisfactory in relieving symptoms. MVD is a surgical treatment method of HFS. Decompression of facial nerve from offending vessel could solve this disorder based on its etiology, and MVD has been established as a highly effective treatment method. MVD for HFS showed an

Received November 16, 2021; Revised November 25, 2021; Accepted November 30, 2021

*Corresponding author: Sang Yeol Yong, Department of Rehabilitation Medicine, Wonju Severance Christian Hospital, Yonsei University Wonju College of Medicine, Wonju 26426, Korea

Tel: +82-33-741-1421, Fax: +82-33-741-1419, E-mail: rehsyyong@yonsei.ac.kr

(C) 2021 Korean Society of Intraoperative Neurophysiological monitoring (KSION)

This is an Open Access article distributed under the terms of the Creative Commons Attribution Non-Commercial License (http://creativecommons.org/licenses/by-nc/ 4.0/) which permits unrestricted non-commercial use, distribution, and reproduction in any medium, provided the original work is properly cited. 
overall effective rate of $91.1 \%-95 \%$ with a mean of follow up of 23.8 months- 2.9 years [1,2]. As the compression is located at the facial root exit zone in $95 \%$, and at the cisternal or the intrameatal portion of the root in $5 \%$, exploration has to be performed through the entire root from the ponto-medullary fissure to the internal auditory meatus [3]. The MVD should be as harmless as possible, because it is a functional surgery. Additionally, if possible, the effect of the procedure should be permanent. This review article aims to review the brainstem auditory evoked potentials (BAEPs) and LSR monitoring, which is frequently implemented intraoperative neurophysiologic monitoring during MVD for safety and effectiveness.

\section{Body}

\section{Brainstem auditory evoked potentials (BAEPs)}

1) Brainstem auditory evoked potentials (BAEPs) and hearing loss

MVD consists of vascular decompression relieving the facial nerve from the offending vessel and implant for the interposition or transposition between the offending vessel and the facial nerve. Offending vessel is usually in the postero-inferior cerebellar artery and not frequently in the antero-inferior cerebellar artery and/or the vertebra-basilar artery [4]. Although the MVD is a safe and effective treatment modality, an important complication of hearing loss may occur. A potential cause of conduction hearing loss is acoustic trauma due to drill noise. Causes of sensorineural hearing loss include direct trauma or stretching of cranial nerve VIII during retracting the cerebellum, manipulation of the vessels; labyrinthine artery or antero-inferior cerebellar artery, newly developed compression by implant material [5]. The operator pays attention from the approach direction to the manipulation procedure so that the perforating arteries to the brainstem is not stretched or damaged, and bipolar coagulation is not used to avoid damage caused by heating. Despite these precautions, hearing loss occurs occasionally, although true prevalence is unclear due to reporting is relying on patient-reported subjective hearing loss and/or inadequate perio- perative audiometric test. BAEPs monitoring decreases the rate of healing loss from $7.7 \%-20 \%$ to below the level of $2.3 \%[5,6]$.

\section{2) Brainstem auditory evoked potentials (BAEPs) principle}

Each wave form of the BAEPs is the result of not one but several generators in relatively small size brainstem. This makes it quite difficult to pinpoint the exact generator of each wave form. However, at least one main generator per wave form has been consistently identified based on study of electocochleography and BAEPs, and clinicopathologic correlation. The three most important waveforms are result of action potentials of following anatomic structures: "wave I" result of distal part of the cochlear part of cranial nerve VIII, "wave III" result of lower pons at the level of the superior olivary complex, "wave V" result of lower midbrain [7].

\section{3) Brainstem auditory evoked potentials (BAEPs) protocols}

American Society of Neurophysiological Monitoring and American Clinical Neurophysiology Society (ACNS) recommends a protocol of $5-12 \mathrm{~Hz} / \mathrm{s}$ stimulation rate and 1,000-4,000 averaging [8]. However, since this protocol requires relatively long time to obtain the BAEPs, there is a limitation in protecting the cranial nerve VIII, which may be damaged in a short time. Therefore, many physicians using higher frequency and lower averaging to obtain the reliable BAEPs quickly within short time $[9,10]$. In one study of these protocols used a protocol of $43.9 \mathrm{~Hz} / \mathrm{s}$ stimulation rate and 400 averaging within about 9 seconds [9].

\section{4) Brainstem auditory evoked potentials (BAEPs) significant changes}

ACNS recommend to alert the surgeon when significant changes of BAEPs occur to prevent hearing loss. The significant changes of ACNS are latency prolongation $\geq 1 \mathrm{~ms}$ or $\geq 10 \%$ increase, and decrease of amplitude $\geq 50 \%$ of wave $\mathrm{V}$ when stimulated according to their protocol [10]. In a recent study, the following criteria were suggested as a critical warning 
sign of real-time BAEPs when stimulated with their protocol. Hearing loss was associated with loss of wave $\mathrm{V}$, and latency prolongation of $1 \mathrm{~ms}$ with at least $50 \%$ reduced amplitude, but latency prolongation of 1 ms without reduced amplitude $>50 \%$ was not associated with hearing loss [11].

\section{Lateral spread response (LSR) and predicting the outcome}

\section{1) Lateral spread response (LSR) principle}

In HFS patients, stimulation of one branch of the facial nerve may result in the activation of the facial muscles innervated by other branches of the facial nerve. This response has been termed LSR. The LSR is an abnormal pathologic electrophysiologic response. The exact mechanisms are not clearly proved, but ephatic transmission of lesion site alone or combination with hyperexcitability of motor nucleus is thought to be related to this response.

\section{2) Lateral spread response (LSR) protocol}

The LSR monitoring is consisted of direct stimulation of facial nerve, commonly at zygomatic braches with pulse duration of $0.1-0.2 \mathrm{~ms}$, frequency of $3 \pm$ $1 \mathrm{~Hz}$ and intensity ranging from $1-2 \mathrm{~mA}$ to $5-25 \mathrm{~mA}$, and recording of compound muscle action potentials of following muscles. The recording sites are the mentalis muscle when the zygomatic branch is stimulated, and in the orbicularis oculi muscles when the mandibular branch is stimulated. When the baseline threshold is determined, any stimulation will proceed responses near baseline level at operation field. However, the LSR may disappear before the MVD is performed, for instance, when the dura is opened. It is suggested that stimulation instensity and/or rate should be increased in these circumstances to reestablish the presence of the LSR in these cases.

\section{3) Lateral spread response (LSR) and effectiveness of microvascular decompression (MVD)}

It is controversial to use the LSR disappearance as an evaluation tool for effectiveness of MVD. If the amplitude of LSR decreases, but LSR does not dis- appear when offending vessel is moved off the nerve, it may indicate that there are another offending vesseles. In this situation, when the other vessel is identified and moved off the facial nerve, the LSR disappears [4]. Although there are a few cases, persistent symptoms or recurrence of spasm were noticed even after surgery for reasons of Teflon felt factor and vascular change, as well as inadequate decompression or neglected offending vessels $[12,13]$. In some cases, it may take about 1 year after MVD for HFS disappearance, this phenomenon supports the central mechanism theory of HFS development including facial motor nucleus hyperexcitability [4]. Despite its modest sensitivity, monitoring of this LSR is performed to guide the operator during surgery because it shows high specificity [14]. Persistent response carries a high risk of persistent hemifacial spasm. The disappearance of LSR during MVD can be a useful tool to predict resolution of symptom shortly after surgery [15]. Although LSR monitoring has limited value in predicting prognosis, it appears to have favorable outcome in short-term period, on the other hand, it does not appear to be effective in predicting the outcome in long-term period $[14,15]$.

\section{Conclusion}

Patients undergoing MVD for HFS should have BAEPs monitoring to prevent hearing loss. Researches on BAEPs has enabled near real-time and safe intraoperative neurophysiologic monitoring, as warning indicators and advanced techniques to get results faster has been discovered. Standardized routine perioperative audiometric test is required to evaluate the true prevalence of hearing loss related to MVD and beneficial verification of BAEPs.

The LSR monitoring is performed to guide the operator during MVD. The disappearance of LSR during MVD appears to be not effective in predicting longterm outcomes, but it demonstrated limited prognostic values in predicting favorable short-term outcomes. Based on these results, although it is difficult to consider the use of LSR alone for intraoperative monitoring during MVD due to the low sensitivity of LSR, 
it may have certain benefits in providing considerable value for investigating the effectiveness of MVD before cranial bone closure.

\section{Ethical approval}

This article does not require IRB/IACUC approval because there are no human and animal participants.

\section{Conflicts of interest}

No potential conflict of interest relevant to this article was reported.

\section{ORCID}

Sang Yeol Yong, https://orcid.org/0000-0003-0288-4121

\section{References}

1. Chung SS, Chang JH, Choi JY, Chang JW, Park YG. Microvascular decompression for hemifacial spasm: a long-term follow-up of 1,169 consecutive cases. Stereotact Funct Neurosurg. 2001;77(1-4):190-3.

2. Miller LE, Miller VM. Safety and effectiveness of microvascular decompression for treatment of hemifacial spasm: a systematic review. Br J Neurosurg. 2012;26(4):438-44.

3. Sindou M, Mercier P. Microvascular decompression for hemifacial spasm: surgical techniques and intraoperative monitoring. Neurochirurgie. 2018;64(2): 133-43.

4. Fernández-Conejero I, Ulkatan S, Sen C, Deletis V. Intra-operative neurophysiology during microvascular decompression for hemifacial spasm. Clin Neurophysiol. 2012;123(1):78-83.

5. Bartindale M, Kircher M, Adams W, Balasubramanian $\mathrm{N}$, Liles J, Bell J, et al. Hearing loss following posterior fossa microvascular decompression: a systematic review. Otolaryngol Head Neck Surg. 2018;158(1):62-75.

6. Sindou M, Mercier P. Microvascular decompression for hemifacial spasm: surgical techniques and intraoperative monitoring. Neurochirurgie. 2018;64(2): 133-43.

7. Simon MV. Neurophysiologic intraoperative monitoring of the vestibulocochlear nerve. J Clin Neurophysiol. 2011;28(6):566-81.

8. American Clinical Neurophysiology Society. Guideline 9C: guidelines on short-latency auditory evoked potentials. J Clin Neurophysiol. 2006;23(2):157-67.

9. Joo BE, Park SK, Cho KR, Kong DS, Seo DW, Park $\mathrm{K}$. Real-time intraoperative monitoring of brainstem auditory evoked potentials during microvascular decompression for hemifacial spasm. J Neurosurg. 2016;125(5):1061-7.

10. Thirumala PD, Carnovale G, Loke Y, Habeych ME, Crammond DJ, Balzer JR, et al. Brainstem auditory evoked potentials' diagnostic accuracy for hearing loss: systematic review and meta-analysis. J Neurol Surg B Skull Base. 2017;78(01):043-051.

11. Park SK, Joo BE, Lee S, Lee JA, Hwang JH, Kong DS, et al. The critical warning sign of real-time brainstem auditory evoked potentials during microvascular decompression for hemifacial spasm. Clin Neurophysiol. 2018;129(5):1097-102.

12. Shu W, Zhu H, Li Y, Liu R. Clinical analysis of repeat microvascular decompression for recurrent hemifacial spasm. Acta Neurol Belg. 2019;119(3):453-9.

13. Lee S, Park SK, Lee JA, Joo BE, Park K. Missed culprits in failed microvascular decompression surgery for hemifacial spasm and clinical outcomes of redo surgery. World Neurosurg. 2019;129:e627-33.

14. Thirumala PD, Altibi AM, Chang R, Saca EE, Iyengar $\mathrm{P}$, Reddy $\mathrm{R}$, et al. The utility of intraoperative lateral spread recording in microvascular decompression for hemifacial spasm: a systematic review and meta-analysis. Neurosurgery. 2020;87(4):E473-E84.

15. Nugroho SW, Perkasa SAH, Gunawan K, Manuhutu YN, Rahman MA, Rizky A. Predicting outcome of hemifacial spasm after microvascular decompression with intraoperative monitoring: a systematic review. Heliyon. 2021;7(2):e06115. 